\title{
Aprendizaje contextualizado y expansivo: Una propuesta para dialogar con las incertidumbres en los procesos educativos
}

Contextualized and expansive learning: A proposal to dialogue with uncertainties in educational processes

\section{Volumen 21, Número 3 \\ Setiembre - Diciembre \\ pp. 1-22}

\author{
Juan Rubio González \\ Taeli Gómez Francisco
}

Citar este documento según modelo APA

Rubio González, Juan. y Gómez Francisco, Taeli. (2021). Aprendizaje contextualizado y expansivo: Una propuesta para dialogar con las incertidumbres en los procesos educativos. Revista Actualidades Investigativas en Educación, 21(3), 1-22. Doi. 10.15517/aie.v21i3.46241 


\title{
Aprendizaje contextualizado y expansivo: Una propuesta para dialogar con las incertidumbres en los procesos educativos
}

\author{
Contextualized and expansive learning: A proposal to dialogue with uncertainties in
} educational processes

\author{
Juan Rubio González ${ }^{1}$ \\ Taeli Gómez Francisco ${ }^{2}$
}

\begin{abstract}
Resumen: A propósito del actual contexto de pandemia, los ámbitos educativos se han visto afectados y tensionados al punto de desarrollarse diversas crisis y exteriorizado las reales condiciones de las prácticas en los centros educativos. En ese contexto, este ensayo científico crítico propone la posibilidad de realizar un diálogo con las incertidumbres en los procesos educativos, a partir de su contextualización y expansión. Lo anterior, implica considerar las tensiones/contradicciones que le son inherentes a estos procesos complejos e histórico-culturales. En ese sentido, se analizaron las problemáticas de los modelos tradicionales de enseñanza, basados en las certezas, predicción y control sustentadas en una matriz epistémica dualista y empirista-positivista, que entraron en crisis en momentos de pandemia. Además, se plantea la necesidad de dialogar con las incertidumbres en los ámbitos educativos, proponiendo la complejidad y el enfoque histórico-cultural como posibles paradigmas mediadores de este proceso. Para sustentar lo anterior se establecieron los principales postulados de las lógicas expansivas y contextualizadas en los procesos educativos, como mecanismos superadores de las certezas y propiciadores de contextos y prácticas educativas que consideren las totalidades, las condiciones materialessociales del contexto cultural, las emergencias, el caos y la diversidad. Se concluye que la matriz epistémica dualista tiende a castrar la enseñanza-aprendizaje y no permite transformaciones, aperturas y diálogos con las nuevas realidades educativas, atributos que sí poseen los procesos educativos contextualizados y expansivos, al ser propuestas heurísticas que permiten comprender y gestionar la complejidad y la incertidumbre de los escenarios actuales.
\end{abstract}

Palabras clave: educación, proceso de aprendizaje, docencia, epistemología.

Abstract: Regarding the current context of pandemic, educational environments have been affected and stressed to the point of developing various crises and externalizing the real conditions of practices in educational centers. In this context, this critical scientific essay proposes the possibility of conducting a dialogue with uncertainties in educational processes, based on its contextualization and expansion. This implies considering the tensions / contradictions that are inherent to these complex and cultural-historical processes. In this sense, the problems of traditional teaching models were analyzed, based on certainties, prediction, and control, supported by a dualist and empiricist-positivist epistemic matrix, which entered into crisis during the pandemic. In addition, the need to dialogue with uncertainties in educational settings was arose, proposing the complexity and the historical-cultural approach as possible mediating paradigms of this process. To support the above, the main postulates of expansive and contextualized logics in educational processes were shown, as mechanisms that overcome certainties and promoters of educational contexts and practices that consider totalities, the material-social conditions of the cultural context, emergencies, chaos and diversity. It is concluded that the dualist epistemic matrix tends to weaken teaching-learning process and does not allow transformations, openings and dialogues with the new educational realities, attributes that contextualized and expansive educational processes do have, as they are heuristic proposals that allow understanding and managing complexity and the uncertainty of current scenarios.

Key words: education, learning processes, teaching profession, epistemology. 1 Universidad de Atacama, Copiapó, Chile. Magister en Investigación Psicológica de la
Universidad de Atacama, Chile. Dirección electrónica: ij.rubio.72@gmail.com Orcid
https://orcid.org/0000-0001-8118-5104

\footnotetext{
${ }^{2}$ Universidad de Atacama, Copiapó, Chile. Doctora en Ciencias Filosóficas de la Universidad de La Habana, Cuba. Dirección electrónica: taeli.gomez@uda.cl Orcid https://orcid.org/0000$\underline{0001-8081-1417}$
}

Ensayo recibido: 16 de marzo, 2021

Enviado a corrección: 4 de junio, 2021

Aprobado: 16 de agosto, 2021 


\section{Introducción}

Los impactos reales en la sociedad provocados no solo por el Covid-19, sino por las estrategias estatales de tratamiento de la pandemia, aún están por verse. Sin embargo, en los ámbitos educativos ya comienzan a aparecer los primeros efectos de estos abordajes y sobre todo, surgen debates sobre las condiciones, muchas veces veladas, en las que se desarrollan los procesos educativos (Murillo y Duk, 2020; Iglesias, González-Patiño, Lalueza y EstebanGuitart, 2020). En esta lógica, se han evidenciado y, por cierto, han generado discusión en este último tiempo factores como como la equidad educativa, el bienestar social y emocional de estudiantes y docentes; el verdadero rol de padres y cuidadores en estos procesos; la motivación y participación del sujeto cognoscente en las nuevas modalidades implementadas, entre otros elementos.

En ese sentido, Bozkurt y Sharma (2020) dan cuenta de cómo la enseñanza remota o a distancia ha sido implementada, mayoritariamente, como una modalidad educativa de emergencia, pero dispuesta de manera precipitada y sin considerar algunos presupuestos básicos. Ante ello, surgen las contradicciones o tensiones internas del proceso educativo, dado que las crisis ahora son manifiestas y no latentes, como en periodos anteriores. Como ejemplo de ello, se reporta que el $90 \%$ de los estudiantes del mundo se han visto afectados por el cierre de escuelas y universidades, lo que ha ampliado las desigualdades de aprendizaje (Unesco, 2020; Unicef, 2020). Por motivos como este, y producto de la actual crisis pandémica, es que emergen con fuerza amplios entornos sociales marcados por la brecha y exclusión social, así como también los fenómenos asociados a ello, que por mucho tiempo permanecieron velados. En ese sentido, las dificultades de conectividad, de equipamiento tecnológico, habitacionales, culturales y educativas (Iglesias et al., 2020) son factores objetivos que no han permitido que se desarrolle óptimamente la digitalización de la enseñanza (Murillo y Duk, 2020) ni que se lleve a cabo, de manera funcional, la modalidad remota.

Sin embargo, concentrarse solo en los déficits o carencias económicas, culturales y sociales, como los factores primordiales de las crisis de los ámbitos educativos, no solo lleva al riesgo de establecer una visión estigmatizadora, sino que representa una mirada parcial del proceso educativo. Ello, porque aparte de lo expuesto hasta aquí, existen elementos de orden epistemológico, paradigmático e inclusive ontológico que están enquistados en la educación actual y que le impiden avanzar hacia estadios que permitan responder a las problemáticas del presente (Castorina, 1994; Morin, 2017). Al respecto, cabe señalar que las epistemologías de base cartesiana utilizadas para comprender la realidad por parte de un sector de la ciencia 
(Bateson, 1998) siguen ancladas en lo que Morin (2017) refiere como la aspiración del ideal de control, orden y de certeza.

Profundizando en lo planteado, una de las definiciones de aprendizaje más universales en la literatura de este ámbito, establece que se trata de "un cambio perdurable en la conducta o en la capacidad de comportarse de cierta manera" (Schunk, 2012, p. 3). En esta definición están implícitas esas aspiraciones de control, orden y certeza a las que se refiere Morin (2017), lo que implica comprender el aprendizaje como el resultado-producto de un conjunto de acciones mecánicas y asociativas, donde los cambios conductuales ocurren tras la manipulación ejercida sobre las condiciones ambientales. Desde esa perspectiva epistemológica y paradigmática de base cartesiana y empírico-positivista, la enseñanza y el aprendizaje se convierten en una dualidad, donde participan, distintivamente, por un lado, docentes poseedores de un conocimiento transmitido como información, y por otro, un sujeto cognoscente dispuesto a recibirlo (Gómez y Rubio, 2017a; Sancho-Asensio et al., 2014).

Así, estas fracturas dualistas y pretensiones de control, orden y certezas corresponden a una realidad en los ámbitos educativos que tienen, al menos, dos repercusiones que impregnan las actuales crisis en tiempos de pandemia. Por un lado, la dualidad centra el proceso educativo en la figura del docente y, por otra, cosifica el contenido a enseñar. Esto tiene como resultado la configuración de un sujeto cognoscente destinado a la repetición y reproducción de materias. Con ello, se reduce la educación a una actividad de procesamiento individualizador destinada a rendir "culto a verdades estáticas, acabadas, al almacenaje de cada vez más información, a la memorización y repetición" (Fernández, 2005, p. 409).

Así mismo, los ideales de control, orden y certezas en el ámbito educativo, han pretendido instalar una lógica de seguridad factual, de verdades eternas, determinismos y actos de fe en la razón (Campos, 2008). Esto se debe a que la educación ha sido entendida como acciones mecánicas y estáticas, donde el movimiento, dinamismo, emergencia e incertidumbre se comprenden como anomalías de un sistema y no como atributos de una realidad compleja. Por ello, Morin (2006) sentencia que "aunque conservemos y descubramos nuevos archipiélagos de certidumbre, debemos saber que navegamos en un océano de incertidumbre" (p. 76). En esa perspectiva, en el ámbito de la educación se configuró una matriz epistémica que estableció una manera particular de conocer, donde se asignaron significados, categorías, leyes y principios (Martínez, 2006) que han definido una dirección que, en este momento, está siendo problematizada desde la teoría y la práctica de los procesos educativos. 
De lo anterior se desprende que no solo los déficits económico, cultural y social son factores relevantes en las crisis de los ámbitos educativos. En la actualidad se pueden observar otros elementos de orden epistémico y paradigmático igual de fundamentales, y que, de ser abordados en su profundidad, generarían cambios cualitativos en este ámbito. Por ello, es relevante poder revisar de manera teórica y práctica estos factores, para comenzar a dar pasos concretos en el abordaje de una educación que dé cuenta de las actuales realidades. Bajo esa lógica, en este trabajo, se propone debatir y problematizar las clásicas matrices epistémicas dualistas y empírico-positivistas que han propiciado ciertos ideales de certeza, predicción y control en el ámbito educativo y que la compleja realidad actual se ha encargado de caracterizar como meras pretensiones o ilusiones.

Desde esa perspectiva, la tesis que se propone en este trabajo viene dada por sostener que desde el paradigma científico de la complejidad y el enfoque histórico-cultural, existen suficientes postulados que permiten desarrollar una posición teórico-práctica que permita alcanzar el diálogo con las incertidumbres en los procesos educativos. Para lo anterior es necesario resituar los procesos educativos, vale decir, comprender que se trata de construcciones integrales y no de resultados parcelados sometidos a una sumatividad artificiosa. De esa manera, un posible diálogo con las incertidumbres implica asumir la cualidad contextual y expansiva de los procesos educativos, lo que precisa conocer los principales rasgos epistémicos que generan sus crisis, así como los posibles abordajes.

\section{Las problemáticas de los modelos tradicionales de enseñanza, que develan las crisis de las certezas en la educación}

Como ya se planteó, las epistemologías y paradigmas sustentadores de los modelos tradicionales de enseñanza han propiciado una matriz epistémica y prácticas pedagógicas marcadas por la dualidad y las certezas. Esto ha permitido que se comprenda la enseñanza y el aprendizaje como dos procesos distintos, operados por sujetos con responsabilidades en la misma lógica. En la práctica, los dos modelos tradicionales, que obedecen a esta lógica dualista, se pueden entender como docencia-enseñanza y docencia-aprendizaje (Rubio, 2021).

El primero, sustentado en el método estructurado de Comenius (1998) y la rigidez como disciplina propiciada por la pedagogía eclesiástica (De Loyola, 1963), a lo que se suma la imbricación con el modelo conductista mediado por el paradigma empirista-positivista, que enfatiza una lógica mecanicista y lineal del funcionamiento psíquico y de los procesos 
educativos. En tanto el segundo, se basa en los postulados de la Escuela Nueva planteados por Jhon Dewey (1957), que pone el foco de los procesos educativos en los intereses y capacidades cognitivas del estudiante, fundamentos que más tarde se nuclean y desarrollan al amparo de modelos etapistas, como el constructivismo cognitivista de raíz piagetana (Piaget, 1968).

Estas concepciones tradicionales configuran un sujeto cognoscente que tiende a acercarse al conocimiento de manera acrítica y neutral. Esto es influido por un tipo de enseñanza que, con el afán de que sea comprendida, tiende a desfragmentar los contenidos en partes desconectadas, absolutizadas y que se enuncian de manera taxativa (Rubio, 2021). Ante esos antecedentes, el sujeto cognoscente solo percibe las sombras de la realidad, siguiendo la lógica platónica de La Caverna: certezas, verdades absolutas, postulados inmodificables y no cuestionables. Esto es lo preocupante, pues los procesos educativos bajo las lógicas tradicionales no favorecen, ni promueven a que el estudiantado se acerque y conecte con la realidad, sino más bien con un reflejo de ella.

Lo anterior se traduce en que la práctica pedagógica sea reducida a la existencia de "un maestro competente que sabe lo que debe aprenderse" (Engeström, 2001, p. 121), lo cual se realiza por medio de acciones con "una pretendida neutralidad” (Gómez y Rubio, 2017a; p. 49) provocando que los sujetos estén "aprendiendo todo el tiempo, algo que no es estable, ni siquiera definido o entendido de antemano" (Engeström, 2001, p. 121). Ante esa problemática se termina responsabilizando a los "los estudiantes por su incapacidad de aplicar contenidos, transferir o generalizar lo aprendido, como también a los docentes se les critica, por su falta de didácticas para transmitir los contenidos" (Gómez y Rubio, 2017a, p. 49).

Este escenario es propiciado debido a que las realidades y prácticas que los sujetos deben enfrentar no presentan las cualidades que la educación tradicional infunde. Por el contrario, la actual pandemia en curso es un claro ejemplo que la realidad posee atributos complejos, de totalidad, de emergencia, de caos, de recursividad y de incertidumbre. En esa lógica, Heisenberg (1958) sentenciaba que "la realidad objetiva se ha evaporado y lo que nosotros observamos no es la naturaleza en sí, sino la naturaleza expuesta a nuestro método de interrogación" (p. 58). Esta interpelación llevada al ámbito educativo no es posible que sea abordada de manera disciplinar, tampoco sumando disciplinas para entender los fenómenos educativos -multidisciplinariedad-; se hacen necesarias modalidades que, a lo menos, sean interdisciplinarias y en lo posible transdisciplinarias, vale decir, abordajes complejos para una realidad que presenta el mismo atributo. 
De lo planteado, se desprende que la realidad actual ha generado un sujeto cognoscente con atributos complejos e integrales, donde la racionalidad objetiva -siguiendo la lógica comtiana- no es, en modo alguno, fuente exclusiva de conocimiento de esa realidad (Osorio, 2007). Así, surge la necesidad de reflexionar sobre praxis educativas que comiencen a considerar las totalidades, las condiciones materiales-sociales del contexto cultural, las emergencias, el caos, las incertidumbres y la diversidad (Gómez y Rubio, 2017b).

A partir de esto se hace necesario responder a la pregunta ¿qué se entiende por incertidumbre? Como primer acercamiento, es conveniente contextualizar que se trata de un constructo que comienza a socializarse desde la Física. En particular, el físico alemán Werner Heinsenberg (1901-1976), en la segunda década del siglo pasado, inserta un razonamiento científico que vendría a revolucionar la teoría cuántica de ese tiempo al establecer la imposibilidad de medir, simultáneamente, de manera precisa y confiable, la posición y el momento lineal de una partícula en el espacio (Lindley, 2008). De lo anterior se desprende que la incertidumbre aparece asociada a conceptos tales como: confusión, inexactitud, indeterminación, entre otros, pero esencialmente, aparece como antagónico a los postulados de certeza, predicción y control que habían dominado la ciencia desde la modernidad.

Lo anterior cobra relevancia en los ámbitos educativos y, en especial, respecto a los procesos de enseñanza-aprendizaje, pues las epistemologías y paradigmas de orden dualista y empírico-positivistas que han primado en ellos han instalado estas concepciones que, a raíz de las diversas crisis sociales que hemos vivido en el último tiempo, se han visto cuestionados y debatidos. No obstante, estos debates no son solo teóricos, sino que, mayoritariamente, provienen desde las propias prácticas que han tenido que desarrollar los protagonistas de estos procesos. En efecto, los ideales, pretensiones e ilusiones de certeza, predicción y control que se habían sostenido como verdades universales e incuestionables por mucho tiempo en el contexto científico y en los procesos educativos, están haciendo crisis en la actualidad.

Desde posturas dualistas son vistos como anomalías del sistema ciertos atributos de la realidad tales como el desorden, el caos, lo impredecible, la emergencia y la incertidumbre. Eso permite explicar la predisposición de estos postulados a poder predecir, manipular y controlar los fenómenos para que retornen al orden y a la normalidad deseados. En la actualidad, se aprecia que tras la imposibilidad de lograr lo anterior, inevitable e inexorablemente emergen las crisis. Estas, que tienen un aparecer marcado por estadios de desconcierto y tensiones generalizadas, fundamentalmente se nutren de dos fenómenos muchas veces imperceptibles: las certezas con que se contaba que, configuradas 
simbólicamente en algunas oportunidades, no permiten dar respuestas funcionales a las nuevas situaciones educativas y exigencias de la nueva realidad. Mientras que los nuevos recursos que emergen o se manifiestan con las crisis, no se pueden reconocer aún o simplemente no están lo suficientemente maduros para ser utilizados.

Lo establecido hace complicado e inapropiado, por decir lo menos, el seguir insistiendo y considerando los ideales y pretensiones de certeza, predicción y control como basamentos válidos para interactuar con los atributos de la realidad actual, sobre todo desde el ámbito educativo. Esta realidad demanda comprender que los fenómenos educativos a los que se debe responder y enfrentar no vienen dados, sino que emergen independiente de la conciencia de los sujetos, lo que obliga a entenderlos como totalidades flexibles, dinámicas, relacionadas y no estables (Lindley, 2008).

Desde esa óptica, las incertidumbres no pueden seguir comprendiéndose como anomalías y fallas de los sistemas educativos, sino más bien, como un atributo esencial de la realidad. Con base en esto, los procesos educativos deben comprenderse de manera contextualizada. De hecho, el mundo como contexto no se mueve a partir de certezas, ni bajo lógicas constantes, regulares, universales o eternas como sostienen los postulados dualistas y empírico-positivistas, por el contrario, las realidades se presentan con atributos de dinamismo, emergencia, caos e inesperadas incertidumbres, difícilmente, sino imposibles de controlar, pretensión e ilusión que el contexto que vive la sociedad actual se ha encargado de develar.

\subsection{La necesidad del diálogo con las incertidumbres en los ámbitos educativos}

Tras lo planteado y ante la imposibilidad de controlar las incertidumbres, solo queda comenzar a desarrollar disposiciones a generar un diálogo con ellas. Lo anterior implica que los procesos educativos deberían ser intencionados hacia las prácticas, más que a configurar una serie de planificaciones y respuestas a priori, que implican un gasto de recursos de todo tipo, que no siempre son recompensados. Eso podría explicar las frustraciones, ansiedades y crisis que, finalmente, se desatan en los ámbitos educativos. Pero disponerse al diálogo con las incertidumbres implica generar problematizaciones de orden epistémico, incluso ontológico, respecto a los postulados que orientan los procesos educativos.

Lo señalado parte por cuestionar la matriz epistémica, la cual sostuvo paradigmas científicos que en la educación han mantenido modelos de acercamiento al conocimiento no coherentes con los contextos actuales. Desde esa perspectiva, las fuentes que originan y 
determinan los métodos, en este caso de enseñanza-aprendizaje, son propios de un periodo histórico-cultural y de una geografía determinada, de ahí la imposibilidad de que sean entendidos como verdades universales. En ese sentido, la matriz epistémica que establecía una base conceptual que propiciaba la objetividad, el determinismo, la cuantificación aleatoria y la verificación empírica de los fenómenos, cuyas fuentes se puede encontrar en el paradigma racionalista y la lógica formal aristotélica, han "confundido el mapa con el territorio, es decir, las palabras o conceptos con la realidad; así manipulando el mapa pensaban manipular la realidad" (Martínez, 2011, p. 137).

En los contextos educativos, estas confusiones e intentos de manipulación de las realidades han propiciado estructuras organizativas esencialmente compartimentadas, donde lo único real y relevante son los métodos que permiten acercarse al conocimiento, que sean coincidentes con los objetivos y necesidades de estas instancias. Esto explica que solo sean validadas las prácticas que se originen de un currículo, programa, pedagogía y didáctica, previamente estandarizado y normado. De esa manera, los ideales de certeza, predicción y control en el ámbito educativo son mecanismos que han propiciado una lógica estanca, reduccionista y con mínimas capacidades de respuestas a los fenómenos de la realidad actual.

Otra característica de la matriz epistémica clásica moderna es que se construye bajo lógicas que postulan una pretendida asepsia y neutralidad en sus procesos. Esto implica que en el contexto educativo se configure un sujeto separado del objeto. Es decir, sujetos poseedores de saberes y otros desprovistos de ellos. Con esto se configuran escenarios educativos con disminuidas capacidades de diálogo, caracterizados por relaciones asimétricas entre quienes transfieren conocimientos a otros que son pasivos, poco participativos y altamente dependientes de un objeto cosificado, como los contenidos transferidos (Gómez y Rubio, 2017a). En esa perspectiva, la dualidad sujeto-objeto también genera una atomización reducida de la realidad y configura pretensiones de existencia de un mundo "inteligible, organizado, predecible, lógico, ordenado, comprensible y reproducible" (Andrade, Cadenas, Pachano, Pereira y Torres, 2002, p. 2).

Lo descrito corresponde a las herencias del racionalismo mecánico que, en términos epistémicos y ontológicos construyeron una realidad que fuese posible controlar y predecir, asociada a una producción de conocimiento construida con base en lógicas de carácter analítico, simple y descriptivo (González Rey, 1997), mientras que la realidad actual obliga a:

(...) aceptar la naturaleza múltiple y diversa de lo estudiado, la integración y desintegración de elementos diferentes y contradictorios en distintos tipos de unidad, la 
aceptación del cambio y la mutabilidad de los objetos, de lo imprevisto como forma de expresión alternativa de un sistema ante hechos similares ocurridos en el tiempo, así como comprender formas irregulares de orden, rompiendo con el concepto de orden equivalente a secuencia regular. (pp. 61-62)

Es decir, la realidad actual obliga a que en los ámbitos educativos se comience a promover rupturas con la matriz epistémica clásica para pasar a adoptar y tener una predisposición a la apertura, flexibilidad, a lo inacabado, lo integral y multivariado, vale decir, hacia una metavisión epistémica multidimensional (Guzmán, 2013). Esto debido a que la educación debe asumir que la realidad actual viene a configurar cosmovisiones complejas, donde se integran la multireferencialidad de los sujetos y objetos que participan en los procesos educativos que, a su vez, desarrollan multiinteracciones y reconstrucciones que también son autogeneradoras de realidad.

\subsection{La complejidad y el enfoque histórico-cultural como paradigmas mediadores para el diálogo con las incertidumbres}

El paradigma científico de la complejidad emerge como antagónico de las lógicas reduccionistas y deterministas de base empírico-positivista que habían prevalecido en el ámbito científico y que habían velado, o siendo más precisos, no tenían los recursos necesarios para reconocer y analizar los atributos de la realidad actual. En ese sentido, ante la lógica mecanicista de comprender los fenómenos del mundo surge la complejidad, buscando "distinguir, reconocer lo singular y lo concreto, sin desunir; religar, en un juego dialógico entre orden, desorden, organización, contexto e incertidumbre, sin dar como verdad esa particular organización de un conjunto determinado" (Andrade et al., 2002, p. 2).

Por su parte, el enfoque histórico-cultural comprende la relevancia de las interacciones sociales en todos los procesos humanos. En ese sentido, la enseñanza-aprendizaje es posible que se desarrolle en la medida que se dé la interacción entre sujetos. Desde esa perspectiva, se entiende que los seres humanos portan recursos de todo tipo que, en esencia, son relaciones sociales interiorizadas, lo cual es posible a partir de la mediación social. Esta corresponde al proceso de transformación del medio que los sujetos realizan utilizando herramientas y mecanismos socialmente construidos (Acosta, 2005), los cuales están permeados de una carga de significados y símbolos histórico y culturalmente definidos, que permiten generar la comunicación y los nexos que propician la integración humana. 
Así, el aprendizaje es fundamental para el desarrollo mental de los sujetos y representa, en esencia, una actividad social y no solo un proceso de realización individual (Vygotsky, 1987). Ante esto, Agramonte y Mena (2006) establecen que la lógica convencional de los procesos educativos de tratar de apartar y no considerar a los sujetos cognoscentes ligados a sus contextos socio-histórico-culturales, equivale a decapitarlos.

De esa manera, la integración y el desarrollo de procesos complejos les son propios a la especie humana, no así la desfragmentación. Por esto, la relevancia de comprender el rol mediador de un docente, no controlador de certezas, pues, en la práctica, debe ser un agente que permite enlazar una trama compleja de relaciones sociales. Así, para lograr que los procesos educativos comiencen a dialogar con las incertidumbres, la enseñanza-aprendizaje debe comprenderse como un proceso que no puede estar focalizado en las partes (docenciaenseñanza, docencia-aprendizaje), sino que debe entenderse y desarrollarse como una relación educativa, a saber, una actividad social integradora y contextualizada (Rubio, 2021).

En ese sentido, el diálogo con las incertidumbres implica que los procesos educativos estén mediados por relaciones educativas más simétricas. Al respecto, Wells (2001) establece que considerar la enseñanza-aprendizaje desde una base dialógica es comprender que "el conocimiento es co-construido conjuntamente [sic] por el enseñante y los estudiantes [sic] mientras realizan actividades conjuntas que se negocian en lugar de ser impuestas" (p. 234). Así, las comunicaciones pedagógicas asertivas constituyen un adecuado clima afectivo en el que se considera la individualidad de los sujetos portadores de historias, significados y símbolos. Estos atributos, en última instancia, son los recursos con que cuenta el sujeto cognoscente y que el mediador del proceso educativo debe transformar en un potencial. Comprender lo anterior, obliga desarrollar una fase que permita gestionar las incertidumbres desde la práctica, no a priori. Ello, debido a que es en el desarrollo de los procesos educativos donde los sujetos que intervienen, descubren y se descubren, construyen y se construyen; el estudiante es objeto-sujeto -a la vez- de su proceso de enseñanza-aprendizaje (Acosta, 2005).

Siguiendo la lógica planteada, los procesos educativos que dialogan con las incertidumbres deben ser disposiciones teórico-prácticas sistémico-complejas, donde los procesos de enseñanza-aprendizaje se desarrollan como sistemas intencionados, articulados y contextualizados, que comprendan la directa relación entre la psiquis, la afectividad, la biografía, el contexto sociocultural y la actividad práctica del sujeto cognoscente (Salas, 2016). Estos antecedentes configuran a los procesos educativos con atributos tales como la imposibilidad de desarrollarse y concretizarse de manera pasiva. Además, con el impedimento 
de entenderlos unívocamente como acciones de orden cognitivo. Esto, pues se trata de actividades sociales esencialmente afectivas, donde el sujeto cognoscente construye y se construye. En esa línea, Wells (2001) establece que "el individuo se transforma en función de su comprensión y su potencial para la acción y, al aplicar estos recursos, también transforma la situación a la que los aplica" (p. 234).

Así, todo proceso educativo y de enseñanza-aprendizaje que intente dialogar con las incertidumbres, debería estar sustentado en prácticas mediadas; co-construcciones que implican la necesaria participación activa de los protagonistas, quienes deben reconocerse insertos en una relación dialéctica objeto-sujeto de transformación y asumiendo el atributo emergente de todos los fenómenos que ocurren en el proceso educativo. En esa lógica, se tiende a cuestionar los postulados explicativos del tipo causa-efecto y estímulo-respuesta (Cole, 2003) tan sobrevalorados por los modelos tradicionales de enseñanza basados en las certezas, pero que en relación al diálogo con las incertidumbres no están aportando.

\subsection{Contextualizar y expandir los procesos educativos; abordando las incertidumbres a partir de las tensiones/contradicciones}

Uno de los planteamientos basales para comprender la incertidumbre en los ámbitos educativos se relaciona con que, en la medida que los sujetos comienzan a mediar con sus contextos, las interacciones van creciendo en complejidad. De esa manera, se constituyen nuevos entornos simbólicos y culturales, imposibles de no ser considerados en los procesos educativos. Estos nuevos intercambios, en términos dialécticos, también generan tensiones o contradicciones en los sistemas, los que a la larga serán el motor que posibilitará los desarrollos y cambios de los procesos educativos.

En ese sentido, las tensiones y/o contradicciones deben comprenderse como parte de los procesos educativos, y estos, a su vez, entendidos como procesos sociales, histórica y culturalmente situados (Díaz, 2003), a saber, que no pueden abstraerse de las situaciones en que se producen. De esto se desprende que la cognición humana es un atributo que emerge en el proceso educativo, "se redistribuye entre formas de actividad conjunta próximas y a distancia y el pensamiento se da tanto entre individuos como dentro de ellos" (Erausquin y Bur, 2017, p. 237). De manera tal que las tensiones/contradicciones siempre están presentes al interior de los sujetos y en las relaciones sociales. De tal forma que son atributos presentes en los procesos cognitivos de interiorización-apropiación de conocimiento y en la exteriorización 
de los procesos psíquicos, orientados a la producción de nuevos artefactos culturales (Cole, 2003; Engeström, 2001).

De manera particular, Engeström (2001) plantea que la interiorización-apropiación y la exteriorización del conocimiento se producen a partir de dinámicas denominadas ciclos expansivos, que surgen y se concretizan en el momento que los sujetos cognoscentes son capaces de cuestionar las prácticas convencionales. Con esto, se provocan movimientos cualitativos en los procesos educativos y se articulan nuevas interacciones producto de la incorporación de las contradicciones/tensiones al sistema educativo. Esto es relevante, pues el conflicto, el cuestionamiento, incluso la insatisfacción, como atributos relacionados con la incertidumbre, se configuran en fuentes e insumos esenciales para desarrollar cualquier proceso educativo, toda vez que de ellos surge la transformación de la práctica (Engeström, 2001).

En esa lógica, hablar de contextualizar y expandir los procesos educativos para abordar las incertidumbres, implica asumir que mediante la interacción social los sujetos, de manera activa, se apropian de prácticas y herramientas culturales. De esa forma entienden e internalizan los símbolos y signos de la cultura y del grupo social al que pertenecen (Gómez y Rubio, 2017a). Pero este proceso está centrado en las contradicciones/tensiones como factores impulsores de movimientos y en las perturbaciones como indicadores del potencial de un sistema/actividad (Engeström, 2001). Es decir, que las cualidades de la incertidumbre, como atributo de la realidad actual, se consideran y organizan para potenciar los procesos educativos.

Estas consideraciones, están basadas en los postulados del Aprendizaje Expansivo (Engeström, 2001) y el Aprendizaje Contextualizado (Gómez y Rubio, 2017a), dos propuestas que consideran la enseñanza-aprendizaje como un proceso dinámico, activo y centrado en experiencias motivantes para los sujetos cognoscentes. Lo anterior, a su vez, está sustentado en el planteamiento vygotskyano de la mediación social en los procesos de enseñanzaaprendizaje. Es decir, en la lógica de que en los procesos educativos los sujetos activos se interrelacionan con los objetos de cognición a través de herramientas e instrumentos mediadores de la interacción (Vygotsky, 1978). Ello se materializa en el modelo triangular denominado acto complejo y mediado, conformado por la tríada sujeto, objeto y artefacto mediador. Elementos que están en permanente y dialéctica interacción y en consideración consciente de la presencia e implicancias de las otras partes de la tríada. 
Al insertarse los artefactos culturales como mediadores de las acciones humanas, ya no es posible seguir considerando al sujeto cognoscente sin su medio cultural (Engeström, 2001). Este antecedente es especialmente significativo al intentar dialogar con las incertidumbres, pues al sujeto ya no se le puede analizar al margen del contexto. Lo mismo ocurre con los objetos, que también deben configurarse como entidades culturales en completa articulación con los sujetos y las comunidades educativas. Estas últimas, a su vez, portan un conjunto de contradicciones/tensiones donde destacan las diversidades culturales, las cuales representan un desafío para los procesos educativos. Estos elementos señalados obligan a considerar la complejidad y multiplicidad de saberes, tradiciones y perspectivas a desarrollar en la enseñanza-aprendizaje y, que de paso, insertan la contradicción/tensión como fuerza impulsora del proceso (Cole, 2003; Wertsch, 1993).

Tras lo señalado, el diálogo con la incertidumbre debe considerar todos estos factores; no puede pretender ignorarlos. De hecho, Engeström (2001) realiza una conceptualización de estos diálogos en los procesos de enseñanza-aprendizaje, indicando que se deben desarrollar:

(...) mediante una constante negociación, orquestación y lucha entre las distintas metas y perspectivas de los participantes ... son como un mosaico en constante evolución, una pauta que nunca se establece por completo ... es un proceso dialogal y en colaboración, donde distintas perspectivas ... y voces ... se encuentran, chocan y se fusionan. (p. 116)

Tras lo analizado, las diversidades culturales surgen como una de las principales incertidumbres de los procesos educativos con las que se debe comenzar a dialogar. Lo anterior, implica comprender que cada vez que la cultura se disocia de los procesos educativos, se produce una descontextualización antinatura que los distorsiona, provocando una castración del aprendizaje y la imposibilidad de generar un diálogo heurístico con estos elementos emergentes. Al respecto, Álvarez y Del Río (1990) señalan que:

(...) la descontextualización instrumental de los aprendizajes que favorece la formación de conceptos, ha ido acompañada de una no tan conveniente descontextualización social cuya magnitud y consecuencias estamos tardando demasiado en evaluar y en atajar desde las disciplinas que se ocupan de la educación como objeto de estudio. (p. 245)

En esa lógica, el contextualizar los procesos educativos, no implica solo considerar los entornos del sujeto y del objeto, pues como lo señala Cole (2003) lo contextual no solo los 
rodea sino que son aquellos atributos que los entrelazan de manera compleja. En ese sentido, Cole (2003) establece que:

Cuando se piensa en el contexto de esta manera, no se puede reducir a aquello que rodea. El contexto es, por el contrario, una relación cualitativa entre un mínimo de dos entidades analíticas (los hilos), que son dos momentos en un único proceso. Los límites entre 'la tarea y su contexto' no son claros y estáticos, sino ambiguos y dinámicos. Por regla general, lo que se toma como objeto y lo que se toma como aquello-que-rodeaal-objeto se constituyen por el acto mismo de nombrarlos. (p. 129)

Esta fundamentación desarrollada por Cole (2003) es relevante para comprender y desarrollar diálogos con las incertidumbres en los procesos de enseñanza-aprendizaje. Esto, pues lo contextual es parte inherente de todo el proceso educativo; sino se considerara de esa manera, se estaría frente a simples acciones de adiestramiento mecánico. Cole (2003) explica lo anterior de la siguiente forma: "(...) un acto en su contexto entendido en términos de la metáfora del entrelazamiento requiere una interpretación relacional de la mente; los objetos y los contextos se presentan juntos como parte de un único proceso bio-socio-cultural de desarrollo" (p. 129).

La explicación de lo planteado, se sustenta en que los sujetos y objetos de un sistema educativo no están disociados de este, sino más bien, están definidos a partir de las relaciones que se dan en el sistema. Por ese motivo, es que el foco para dialogar con las incertidumbres debe estar puesto en esa relación y en la dirección o sentido que ésta va adquiriendo; solo así se podrá dialogar desde las prácticas y dejar de pretender controlar los procesos educativos. En ese sentido, Bateson (1998) señala que sujetos y objetos no pueden ser recortados de la realidad que les circunda. En esa lógica, lo contextual es un atributo de la realidad; son las conexiones complejas entre lo ambiental, los sujetos y objetos. En la práctica, no existe una desfragmentación o atomización de los sujetos, objetos y su contexto. Por el contrario, existe una interconexión dialéctica y dinámica, que imposibilita muchas veces, visualizar los límites entre ellos, de ahí la relevancia por reconocer esos entrelazamientos inciertos para comenzar a dialogar con ellos.

Configurando lo anterior, en los procesos educativos y su diálogo con las incertidumbres, se podría señalar que el docente es un mediador de posibles vías que permitan conectar e intencionar procesos, didácticas o metodologías. Desde esa configuración, tanto él, como el estudiante, son elementos llamados a establecer una relación educativa compleja. Que lo es, 
no porque sea dificultosa realizar, sino porque se trata de un entramado integral, dialógico, dinámico y contextualizado a la realidad histórico-cultural donde se produce el proceso educativo. En ese sentido, un contexto de enseñanza-aprendizaje, no es algo dado, sino que son co-construcciones definidas por las relaciones educativas dadas por los sujetos que intervienen. Al respecto, Lacasa y Silvestri (2001) señalan que:

(...) los contextos no han de entenderse como algo definitivamente dado, sino que se constituyen dinámicamente, mutuamente con la actividad de los participantes. Un contexto se delimita por lo que la gente hace, dónde y cuándo lo hace y, en este sentido, las personas que interactúan llegan a constituir el contexto para los demás (pp. 340341).

En esa perspectiva, los procesos educativos contextualizados buscan desarrollar prácticas sustentadas en la actividad social de los sujetos y requieren de estrategias y didácticas psicoeducativas tendientes a promover la reflexión en la acción de éstos (Gómez y Rubio, 2017a). Esto se explica porque las incertidumbres están presentes y se puede dialogar con ellas desde la práctica, incentivando, además, procesos educativos colectivos y cooperativos. Es decir, atmosferas dialogantes, discusiones grupales destinadas a promover participación y vinculación de los protagonistas del proceso de enseñanza-aprendizaje (Gómez y Rubio, 2017a). A lo que se debería agregar la co-construcción de las evaluaciones del proceso educativo, dado que los sujetos involucrados deben comprometerse en todo este entramado pedagógico.

Así, la contextualización de los procesos educativos, permitirá dialogar con las incertidumbres, ya que generará ámbitos "dinámicos, activos y centrados en experiencias motivantes para los protagonistas" (Gómez y Rubio, 2017a, p. 52). En esa lógica, deben surgir ciertas transformaciones epistémicas y prácticas. De partida, los procesos educativos no pueden desarrollarse exclusivamente en el espacio físico de aulas estancas y compartimentadas, pues, claramente las experiencias desarrolladas en el contexto de la pandemia así lo han demostrado, la enseñanza-aprendizaje trasciende fronteras físicas del aula (Gómez y Rubio, 2017b). Además, enfrentar las incertidumbres y dialogar con ellas, obliga a que los procesos educativos no tengan que desarrollar tránsitos secuenciales establecidos en rutas a priori, pues la realidad demuestra que la práctica debe estar orientada por otros factores; el contexto socio-cultural, las particularidades de la comunidad de aprendizaje, el 
conocimiento del docente y las diversidades de estudiantes, atributos, que en última instancia, definirán el tránsito que seguirá el proceso.

Asumiendo que el diálogo con las incertidumbres, debe considerar lo poco conducente que resulta proponer rutas a priori, pues esta debe surgir de la práctica del proceso educativo (Gómez y Rubio, 2017a), lo relevante de la contextualización radica en asumir y desarrollar los conflictos, vale decir, las contradicciones/tensiones internas que se producen en cada momento educativo. Esto, debido a que son estos atributos de la realidad, los que permiten movilizar los procesos educativos y comenzar a dialogar con las incertezas. Para lograrlo, se requiere contar con soportes teóricos y prácticos que apoyen a las comunidades educativas en el desarrollo de estrategias de aprendizaje, didácticas y capacidades cognitivas e interpersonales. Lo anterior implica tener una orientación, a lo menos, interdisciplinaria de los procesos formativos (Gómez y Rubio, 2020).

Así, contextualizar y expandir los procesos educativos, para permitir el diálogo con las incertidumbres, implica asumir la complejidad de la realidad actual, con cambios culturales y sociales emergentes y dinámicos, que obligan a desarrollar nuevas maneras de organizar los procesos de enseñanza-aprendizaje, contemplando los mismos atributos descritos. Desde esa lógica, los sujetos cognoscentes expandirán sus límites culturales y de sus conocimientos. En la práctica, y siguiendo a Engeström (2001) se transformará el repertorio cultural de acciones, que posibilitará arribar a estadios evolutivos que permitan a los sujetos realizar lo mismo, pero de manera más eficaz y eficiente. Eso explica que expandir los procesos educativos permite construir redes y configurar un agregado de sujetos diversos, pero articulados en un colectivo educativo común. En esos contextos, se desarrollan dinámicas caracterizadas por un diálogo más simétrico y asumiendo las contradicciones/tensiones que surjan, pues estas son los factores detonadores y movilizadores que propiciarán poder comprender y dialogar con la realidad emergente.

En ese sentido, expandir y contextualizar los procesos educativos, implica ir más allá del hecho de adquirir saberes de manera personal y buscar competencias en la misma línea. Sino más bien, requiere lograr entrelazar fenómenos que permita al estudiantado abrir nuevas posibilidades de diálogo con la realidad. En esa línea, lograr dialogar con las incertidumbres, implica entender y desarrollar los procesos educativos como una articulación interrelacionada de acciones, conformando redes que mantienen una lógica recursiva, en el que se propician mecanismos de transición a través de contradicciones/tensiones que surgen en la comunidad educativa. 


\section{Conclusiones}

Los últimos acontecimientos ocasionados por la pandemia han movilizado las configuraciones subjetivas de los sujetos y han develado el constante cambio y la poca certeza que existe para relacionarse con las dinámicas de la realidad actual. En el ámbito educativo, han revivido las viejas preguntas y dudas sobre qué y para qué educar. Las respuestas, nuevamente, parecen no estar claras, de ahí el surgimiento de desconcierto en ámbitos donde las crisis parecen ser realidades constantes. Sin embargo, estas tensiones/contradicciones también permiten la emergencia de problematizaciones teóricas y prácticas necesarias. Así, el trabajo desarrollado se ha propuesto debatir con las clásicas matrices epistémicas que, en el ámbito de la educación, han propiciado ciertos ideales de certeza, predicción y control que la realidad se ha encargado de caracterizar como pretensiones o ilusiones.

Desde esa perspectiva, el trabajo pretende abrir un espacio que permita dialogar con las incertidumbres del mundo actual. Así, se propone la teoría científica de la complejidad y el enfoque histórico-cultural como posibles paradigmas movilizadores que orienten la posibilidad de comenzar a dialogar con los atributos de la realidad, que no presenta principios únicos y estables sobre los cuales fundamentar acciones a priori. Ello, como consecuencia de que la matriz epistémica mecánica, lineal, reduccionista y determinista pareciera no dar respuestas a fenómenos emergentes como la incertidumbre.

En ese sentido, emerge el cuestionamiento a las lógicas focalizadas en las partes del proceso educativo que, por su carácter reduccionista y fragmentador, estancan y, en parte, tienden a castrar la enseñanza-aprendizaje al no permitir transformaciones, aperturas ni diálogos con las nuevas realidades. A partir de esto, Engeström (2001) concluye que las "teorías de aprendizaje estándar tienen poco que ofrecer, si uno quiere comprender estos procesos" (p. 121). En esa lógica, la complejidad y el enfoque histórico-cultural realizan propuestas heurísticas que permiten comprender la integralidad de los procesos educativos. Así mismo, establecen formas para entender el rol de los contextos, las particularidades del sujeto cognoscente, la cultura, las mediaciones sociales, elementos y factores que no pueden ser omitidos de estos análisis, pues son parte de toda actividad humana y permiten el surgimiento de la conciencia de los sujetos y el desarrollo de sus potencialidades (Kozulin, 2002).

En esa línea, el diálogo con las incertidumbres en los ámbitos educativos implica comprender como punto de partida que toda acción humana se desarrolla en un sistema social y cultural, determinado y complejo. Por eso la imposibilidad de descontextualizar los procesos 
educativos. A lo anterior se suma el atributo de que los sujetos cognoscentes son portadores de historias y propósitos, por lo cual es inherente en ellos el poder potenciar sus recursos. Por los antecedentes señalados, surge la necesidad de que los procesos educativos deban ser contextualizados, pero también expansivos. Toda vez que esas cualidades permitirán que los sujetos alcancen su mayor potencial y a medida que lo logren, tendrán mayores recursos para dialogar con un mundo siempre incierto y emergente.

Así, los procesos educativos al ser contextualizados y expansivos no pueden configurarse como construcciones teóricas alejadas de la realidad, sino que deben construirse insertos en las prácticas. Lo señalado, precisa considerar la diversidad de instrumentos simbólicos y materiales que intervienen en los procesos educativos donde se entrecruzan los aspectos biológicos, psicológicos, cognitivos, socio-históricos y culturales (Salas, 2016). Desde aquí, la relevancia de entenderlos como un sistema de actividades sociales complejas, integrales y dinámicas.

En atención a lo planteado, en este trabajo se hace una invitación a cuestionar las matrices epistémicas tradicionales, centradas en las partes, donde los conocimientos del sujeto cognoscente aparecen disociados o de manera independiente de sus prácticas sociales. Con esto se provoca una evidente ruptura entre el qué saber y el cómo saber (Gómez y Rubio, 2017a). En esa lógica, el contextualizar y expandir para dialogar con las incertidumbres, implica gestionar desde lo abstracto a lo concreto, además, aceptar y movilizar las críticas y las tensiones/contradicciones internas de los procesos.

Considerando estas cualidades, impulsar una relación certera con la realidad actual obliga a que los procesos educativos estén orientados y abordados interdisciplinaria o transdisciplinariamente. Esto, para propiciar diálogos disciplinares que permitan visualizar, analizar y construir nuevas ideas y soluciones a los procesos de enseñanza-aprendizaje. Pero esta vez, no desde linealidades metodológicas, planificadas a priori, sino considerando el contexto histórico-cultural. En definitiva, desarrollando un diálogo que se haga cargo de los nudos teórico-prácticos que la realidad actual obliga a resolver con urgencia, ya que la matriz epistémica basada en las certezas y el control no ha podido dar esas respuestas.

\section{Referencias}

Acosta, María. (2005). Tendencias pedagógicas contemporáneas: La pedagogía tradicional y el enfoque histórico-cultural. Análisis comparativo. Revista Cubana de Estomatología, 42(1). Recuperado de http://scielo.sld.cu/scielo.php?script=sci arttext\&pid=S0034-

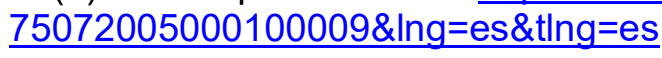


Agramonte, Alain. y Mena, Francisco. (2006). Enfoque Histórico Cultural y de la Actividad en la formación del licenciado en enfermería. Revista Cubana de Enfermería, 22(2). Recuperado de http://scielo.sld.cu/scielo.php?script=sci arttext\&pid=S086403192006000200006\&lng $=$ es\&tlng=es

Álvarez, Amelia. y Del Río, Pablo. (1990). Educación y desarrollo: la teoría de Vygotsky y la Zona de Desarrollo Próximo. En C. Coll, J. Palacios y A. Marchesi (Comps.), Desarrollo psicológico y educación (vol. II; pp. 93-119). Madrid: Alianza.

Andrade, Raiza., Cadenas, Evelin., Pachano, Eduardo., Pereira, Luz. y Torres, Aura. (2002). El paradigma complejo: un cadáver exquisito. Cinta de Moebio, 14, 236-279. Recuperado de https://cintademoebio.uchile.cl/index.php/CDM/article/download/26232/27524/0

Bateson, Gregory. (1998). Pasos hacia una ecología de la mente. Buenos Aires, Argentina: Editorial LOHLÉ-LUMEN.

Bozkurt, Aras. y Sharma, Ramesh. (2020). Emergency remote teaching in a time of global crisis due to coronavirus pandemic. Asian Journal of Distance Education, 15(1), 1-6. doi: https://doi.org/10.5281/zenodo.3778083

Campos, Rodrigo. (2008). Incertidumbre y complejidad: reflexiones acerca de los retos y dilemas de la pedagogía contemporánea. Revista Actualidades Investigativas en Educación, 8(1). doi: https://doi.org/10.15517/aie.v8i1.9326

Castorina, José. (1994). Problemas epistemológicos de las teorías del aprendizaje en su transferencia a la educación. Perfiles Educativos, (65), 3-16. Recuperado de https://www.redalyc.org/articulo.oa?id=13206501

Cole, Michael. (2003). Psicología cultural. Una disciplina del pasado y del futuro. Madrid, España: Morata.

Comenius, Juan. (1998). Didáctica Magna. México: Porrúa.

Dewey, Jhon. (1957). La educación de hoy. Buenos Aires, Argentina: Losada

Díaz, Frida. (2003). Cognición situada y estrategias para el aprendizaje significativo. Revista Electrónica de Investigación Educativa, 5(2), 105-117. Recuperado de https://redie.uabc.mx/redie/article/view/85/151

Engeström, Yrjö. (2001). Expansive Learning at Work: Toward an activity theoretical reconceptualization. Journal of Education and Work, 14(1), 133-156. doi: https://doi.org/10.1080/13639080020028747

Erausquin, Cristina. y Bur, Ricardo. (2017). Psicólogos en contextos educativos (2a ed.). Buenos Aires, Argentina: PsiDispa.

Fernández, Lourdes. (2005). Trabajar en grupos en los contextos educativos. En M. Alejandro y M. Romero (Comps.), Trabajo grupal y coordinación: Selección de lecturas (pp. 405414). La Habana, Cuba: Caminos. 
Gómez, Taeli. y Rubio, Juan. (2017a). Cognición contextualizada: Una propuesta didáctica y psicopedagógica socioconstructivista para la enseñanza-aprendizaje del derecho. Revista Pedagogía Universitaria y Didáctica del Derecho, 4(2), 40-63. doi: https://doi.org/10.5354/0719-5885.2017.47970

Gómez, Taeli. y Rubio, Juan. (2017b). Complejidad-educación: un aporte para las generaciones futuras. Revista Educación y Humanismo, 19(33), 409-423. doi: https://doi.org/10.17081/eduhum.19.33.2653

Gómez, Taeli. y Rubio, Juan. (2020). Enseñanza-aprendizaje universitaria integral: una propuesta educativa, basada en el paradigma de la complejidad y el enfoque históricocultural. Chile: Editorial Periferias.

González-Rey, Fernando. (1997). Epistemología Cualitativa y Subjetividad. La Habana, Cuba: Pueblo y Educación.

Guzmán, Jean. (2013). Metódica para abordaje una investigación desde una perspectiva transcompleja. Revista de Investigación, 37(79), 13-32. Recuperado de http://ve.scielo.org/scielo.php?script=sci arttext\&pid=S1010$\underline{29142013000200002 \& \operatorname{lng}=e s \& t \operatorname{lng}=e s}$

Heisenberg, Werner. (1958). Physics and philosophy: the revolution of modern science. Nueva York: Harper \& Row.

Iglesias, Edgar., González-Patiño, Javier., Lalueza, José. y Esteban-Guitart, Moisés. (2020). Manifiesto en Tiempos de Pandemia: Por una Educación Crítica, Intergeneracional, Sostenible y Comunitaria. Revista Internacional de Educación para la Justicia Social, 9(3), 181-198. doi: https://doi.org/10.15366/riejs2020.9.3.010

Kozulin, Alex. (2002). Sociocultural theory and the mediated learning experience. School Psychology International, 23(1), 7-35. doi: https://doi.org/10.1177/0143034302023001729

Lacasa, Pilar. y Silvestri, Adriana. (2001). Contextos de aprendizaje y desarrollo. Una mirada desde Latinoamérica. Cultura y Educación, 13(4), 339-354. doi: https://doi.org/10.1174/113564001753366739

Lindley, David. (2008). Incertidumbre. España: Editorial Ariel.

Loyola, Ignacio De. (1963). Obras Completas (2 ${ }^{\mathrm{a}}$ ed.). Madrid, España: Editorial Católica S.A.

Martínez, Miguel. (2006). Conocimiento Científico General y Conocimiento Ordinario. Cinta Moebio (27), 1-10. Recuperado de https://www.redalyc.org/articulo.oa?id=101/10102701

Martínez, Miguel. (2011). El "Saber Pensar" en la investigación y sus principios. Espacio Abierto, 20(1), 131-157. Recuperado de https://www.redalyc.org/pdf/122/12218314006.pdf

Morin, Edgar. (2006). El método 6. Ética. Madrid, España: Cátedra 
Morin, Edgar. (2017). El Método 1. La naturaleza de la naturaleza. Madrid, España: Ediciones Cátedra.

Murillo, Francisco. y Duk, Cynthia. (2020). El Covid-19 y las brechas educativas. Revista Latinoamericana de Educación Inclusiva, 14(1), 11-13. Recuperado de https://scielo.conicyt.cl/pdf/rlei/v14n1/0718-7378-rlei-14-01-11.pdf

Osorio, Francisco. (Ed.). (2007). Epistemologías de las ciencias sociales. Breve manual. Santiago de Chile, Chile: Ediciones UCSH.

Piaget, Jean. (1968). Educación e instrucción. Buenos Aires, Argentina: Proteo.

Rubio, Juan. (2021). Desafíos para la docencia universitaria. Una propuesta desde el enfoque histórico-cultural. México: Editorial LEED [En prensa].

Salas, Flora. (2016). Aportes del modelo de Yrjö Engeström al desarrollo teórico de la docencia Universitaria. Revista Educación, 40(2), 1-22. doi: https://doi.org/10.15517/revedu.v40i2.15257

Sancho-Asensio, Andreu., Solé, Xavi., Montero, José., Navarro, Joan., Canaleta, Xavi. y Vernet, David. (2014). Support tool for the formation of working groups in collaborative learning environments. 9th Iberian Conference on Information Systems and Technologies (CISTI). Barcelona, España. https://doi.org/10.1109/CISTI.2014.6876942

Schunk, Dale. (2012). Teorías del aprendizaje. Una perspectiva educativa (6a ed.). México: Pearson Educación

Unesco. (2020). COVID-19 Education Response. Recuperado de https://en.unesco.org/covid19/educationresponse/globalcoalition

Unicef. (2020). UNICEF and Microsoft launch global learning platform to help address COVID19 education crisis. Recuperado de https://www.ndtv.com/education/unicef-microsoftlaunch-global-learning-platform-to-address-covid-19-education-crisis-2216245

Vygotsky, Lev. (1978). Mind and Society. Cambridge, MA: Harvard Press University.

Vygotsky, Lev. (1987). Historia del desarrollo de las funciones psíquicas superiores. La Habana, Cuba: Ed. Científico.

Wells, Gordon. (2001). Indagación dialógica. Hacia una teoría y una práctica socioculturales de la educación. Barcelona, España: Paidós.

Wertsch, James. (1993). Voices of the Mind: Sociocultural Approach to Mediated Action. Cambridge, MA: Harvard University Press. 


\section{Revista indizada en}
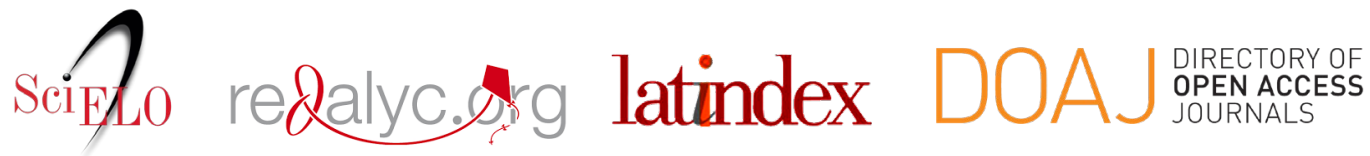

Distribuida en las bases de datos:
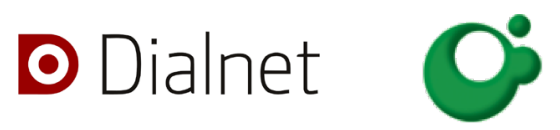
SHERPA/RøMEO

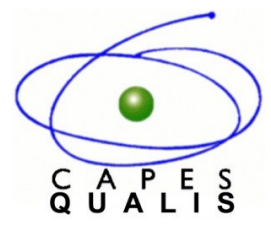

MIAR 